Once again, water, or the lack thereof, is thought to be responsible for this planetary contrast in tectonic strength. Recent high-temperature laboratory experiments on the creep strength of rocks that have been thoroughly dried demonstrate that the presence of even a small amount of water profoundly decreases the strength of crustal rocks 9 . Only as the experimentalists have begun simulating conditions on Venus have rock samples been dried thoroughly enough to detect this sensitivity to small amounts of water. It appears that the very hot, dry conditions of Venus are essential in preserving the strength required to build and support her high-relief mountains.

Sean Willett is in the Department of Geosciences, The Pennsylvania State University, 503 Deike Building, University Park, Pennsylvania 16802, USA.

\title{
Accounting for light-element abundances
}

\section{A. G. W. Cameron}

LAST year's discovery ${ }^{1}$ of gamma-rays from the Orion star-forming region that were attributable to de-excitation of carbon and oxygen has far-reaching consequences, as it implies an unexpectedly high flux of low-energy cosmic rays in the region. Astrophysicists initially welcomed this as a possible explanation for the high abundances of radioactive nuclides in the interstellar medium ${ }^{2,3}$, but on page 318 of this issue, Cassé, Lehoucq and Vangioni-Flam ${ }^{4}$ suggest that it could account for the cosmic abundances of the light elements lithium, beryllium and boron.

Because the observed $\gamma$-ray lines are broad, it is evident that the $\gamma$-rays are emitted from energetic particles, thus indicating a flux of relatively low-energy cosmic rays some 30 times that expected near the Sun (the particles are not detected from the Earth, so they must lie below the low-energy cut-off). At least in the larger star-forming regions, including Orion, supernova explosions will be produced in the more massive stars, and these are natural sources of this extra component of lower-energy cosmic rays. So the excess cosmic rays will be concentrated within such star-forming regions, as it will take some time for them to diffuse out of the local complex tangle of magnetic fields.

Clayton ${ }^{2}$ immediately seized upon this excess flux to suggest that the large amount of the radioactive isotope ${ }^{26} \mathrm{Al}$ in the interstellar medium could have been produced in star-forming regions in this way, and that this and other extinct radioactivities in the early Solar System might have been produced by energetic particle bombardment in the fragment of an interstellar molecular cloud that collapsed to form the Sun. He assumed that a solar-like abundance spectrum of particles had been accelerated in Orion. Such a spectrum, though, would have produced detectable $\gamma$-rays in the 1 - to $3-\mathrm{MeV}$ region, and these were not seen. Clayton has therefore retracted his suggestion about the production of ${ }^{26} \mathrm{Al}$ in the interstellar medium but stands by a modified form of his suggestion concerning the extinct radioactivities in the early Solar System. In my view some such bombardment process seems necessary to explain the presence in the early Solar System of the short-lived nuclide ${ }^{41} \mathrm{Ca}$ (ref. 3 ).

Now Cassé, Lehoucq and VangioniFlam $^{4}$ have applied our new-found knowledge about the Orion region to the question of the abundances of $\mathrm{Li}, \mathrm{Be}$ and $\mathrm{B}$. They start with a large helping of common sense. Because it is the massive stars in the Orion OB1 association that have produced many supernovae and a large interstellar superbubble extending from Orion to Eridanus ${ }^{5}$, then the energetic particles that were accelerated should reflect the composition of the massive envelopes of these stars before their supernova stage. In the pre-supernova evolution and in the explosions themselves, large amounts of helium, carbon and oxygen were synthesized, and therefore it is a reasonable expectation that these elements should dominate in the compositions of the excess low-energy cosmic rays that were accelerated in the aftermath of the explosions. In a symposium last year on the evolution of star-forming regions*, R. Ramaty emphasized that the observed spectrum of Orion $\gamma$-rays required carbon and oxygen acceleration but, to minimize the production of 1 - to $3-\mathrm{MeV} \gamma$-rays, relatively little acceleration of still heavier elements and of hydrogen.

It has long been recognized that $\mathrm{Li}, \mathrm{Be}$ and $B$ are not products of stellar nucleosynthesis in the same way that nearly all the other elements are, and so the standard explanation for their presence in nature has been to attribute their production to cosmic-ray spallation - the shattering of nuclei through collisions - in the

*Workshop on the Evolution of Star-Forming Regions, Center for Astrophysics, Cambridge, Massachusetts, USA, 24-26 October 1994. interstellar medium (with an excess amount of ${ }^{7} \mathrm{Li}$ attributed to primordial nucleosynthesis). However, if one uses a cosmic-ray energy spectrum given by an inverse power law and known spallation cross-sections with known interstellar medium abundances, straightforward calculations fail to reproduce the relative isotope abundances of these light elements. In general, there is a problem with boron: the $\mathrm{B} / \mathrm{Be}$ ratio is roughly double the current ratio for stellar sources formed in the early galaxy, and the current ${ }^{11} \mathrm{~B} /{ }^{10} \mathrm{~B}$ ratio is nearly twice as great as the calculation predicts ${ }^{6}$.

It was recognized ${ }^{6}$ in 1971 that these ratios could be accounted for if an extra source of low-energy cosmic rays could exist above that predicted by the above power-law spectrum, for these would not be directly observable from the Earth. In that case there would be an excess production of ${ }^{11} \mathrm{~B}$ relative to ${ }^{10} \mathrm{~B}$ owing to the difference in the energy thresholds for the production. Cassé et al. have now shown that the excess flux of low-energy cosmic rays in Orion provides a natural explanation for these long-standing discrepancies, and that a plausible galactic evolution calculation can fit the abundances nicely.

There is an interesting footnote to this story. The possibility that the energetic particles in Orion-like regions could produce the missing boron has also been considered by Fields et al. ${ }^{7}$, who tentatively reject this idea because they expect that the accelerated particles would produce too many $1-3-\mathrm{MeV} \gamma$-rays and that a universal low-energy flux would produce too much ionization of the interstellar medium. They suggest instead that the additional ${ }^{11} \mathrm{~B}$ might be produced by neutrino-induced reactions in supernova explosions. Cassé et al. avoid these difficulties by proposing that the Orion flux has a composition similar to the envelope abundances produced in a massive supernova explosion, and that the flux is concentrated in star-forming molecular clouds and does not characterize the flux in the general interstellar medium. Many aspects of the Orion puzzle remain unclear, and I expect that there will be more theoretical surprises before the dust settles.

A. G. W. Cameron is in the HarvardSmithsonian Center for Astrophysics, 60 Garden Street, Cambridge, Massachusetts 02138, USA.

\footnotetext{
1. Bloemen, H etal Astr. Astrophys, 281, L5-L8 (1994)

Clayton, D. D. Nature 368, 222-224 (1994)

3. Cameron, A. G. W. Meteoritics (in the press).

4. Cassé, M., Lehoucq, R. \& Vangioni-Flam, E. Nature 373 318-319 (1995)

5. Burrows, D. N. et al. Astrophys. J. 406, 97-111 (1993).

6. Meneguzzi, M. Audouze, J. \& Reeves, H. Astr. Astrophys. 15, 337-359(1971)

7. Fields, B. D. Olive, K. A. \& Schramm, D. N. Astrophys. J. (in the press)
} 